\title{
Marketing territorial et promotion de la ville de Casablanca
}

\section{Territorial marketing and promotion of the city of Casablanca}

\author{
Maha BENDAHMANE \\ Université Mohammed V, Rabat, Maroc
}

\begin{abstract}
Résumé : Cet article sera consacré à l'étude de l'utilité du Marketing territorial, et sa contribution dans la promotion du territoire de Casablanca. La réalisation de notre objectif a imposé la mobilisation d'une étude qualitative qui consiste à analyser des guides d'entretien adressés à l'ensemble des autorités territoriales à l'aide de l'outil « Nvivo ». Par ailleurs, l'analyse de ces guides d'entretien adressés aux différents acteurs du territoire de Casablanca, a montré que certes la création de la marque territoriale «Wecasablanca» est récente et donc les impacts ne peuvent être mesurés dans l'immédiat, mais la démarche est fédératrice, et actuellement avec la pratique du marketing territorial comme étant un outil majeur pour la ville qui est devenue la troisième position des villes les plus attractives pour les investissements en Afrique et dans le Moyen-Orient, la 3eme destination touristique nationale avec le tourisme d'affaires, néanmoins son attractivité nationale et internationale la confronte à plusieurs défis. En effet, le marketing territorial présente un outil parmi d'autres outils qui contribue à la promotion du territoire.
\end{abstract}

Mots-clés : marketing territorial, promotion territorial, marque territoriale, offre territoriale.

\section{Introduction}

Il est essentiel pour un territoire d'avoir une bonne image à l'extérieur pour attirer de nouveaux habitants, plus de touristes et d'investisseurs, ainsi qu'à l'intérieur afin de générer auprès de ses habitants un sentiment d'attachement. Sauf qu'un territoire pour qu'il soit attractif, il doit disposer notamment d'un avantageux environnement économique, législatif, et social; d'une part de marché territoriale considérable ; des interlocuteurs territoriaux de qualité ; et des traits distinctifs du territoire, à savoir son image, ses atouts et potentialités.

La forte concurrence qui caractérise les territoires ainsi que les nouveaux enjeux de l'attractivité ont poussé à s'intéresser beaucoup plus au Marketing territorial qui est considéré comme étant un ensemble de techniques permettant de promouvoir l'attractivité du territoire et d'avoir une offre territoriale répondant aux attentes des consommateurs. Cette forme de gestion aidera les institutions 
concernées à prendre en considération les forces et les faiblesses du territoire, et à communiquer plus efficacement pour offrir une visibilité supérieure aux utilisateurs du territoire.

Plusieurs territoires ont réalisé de véritables résultats en matière de développement des territoires grâce au marketing territorial, notamment le territoire de Lyon et celui de Berlin qui pratiquent le marketing territorial en créant la marque territoriale « Be Berlin » et « Onlylyon », ce qui a encouragé d'autres territoires notamment marocains à mettre en œuvre des stratégies de marketing territorial. Par ailleurs, le territoire de Casablanca a commencé à pratiquer le marketing territorial à travers la création de la marque territoriale «Wecasablanca» qui vise à renforcer sa notoriété et son image à l'échelle nationale et internationale.

Cette recherche s'articule autour de deux cadres: l'un théorique et l'autre pratique visant tous les deux à comprendre le rôle que joue le marketing territorial dans la promotion des territoires. Par conséquent, nous traduisons notre problématique sous la forme de la question principale suivante à laquelle nous souhaitons apporter une réponse dans le cadre de ce travail de recherche: Peut-on affirmer que la pratique du marketing territorial permet d'assurer la promotion des territoires?

\section{Marketing territorial et promotion des territoires : cadrage théorique}

« Le marketing territorial est l'effort de valorisation des territoires à des marchés concurrentiels pour influencer, en leur faveur, le comportement de leurs publics par une offre dont la valeur perçue est durablement supérieure à celle des concurrents. Cette activité est généralement pilotée par des agences de développement pour le compte d'autorités publiques ou d'acteurs privés » (Gollain, 2008).

Elle est une démarche visant à qualifier l'offre présente sur le territoire et à proximité, en vue de le rendre attractif. Trois métiers composent le marketing territorial, celui de réaliser des études afin de faire un état des lieux précis des atouts du territoire et des opportunités de marché à saisir, celui de développer une stratégie en réponse à la problématique soulevée par l'étude, et celui de la mettre en œuvre par la prospection et la communication (Benabdelhadi, Moussalim, 2012). Ce marketing consiste à mettre le citoyen au centre de toute décision prise au niveau du territoire et développer son implication dans la gestion de la vie de la collectivité locale à laquelle il appartient.

Le marketing territorial constitue un atout concurrentiel qui aidera à faire face à la concurrence, différencier le territoire, le positionner par rapport aux concurrents et créer des attitudes favorables envers un territoire.

\subsection{Les outils du Marketing territorial}

L'application du marketing territorial a pour objectif d'élaborer, sur la base de la connaissance de l'environnement, l'offre territoriale intégrant à la fois des produits et des services destinés soit aux résidents permanents ou/et aux utilisateurs occasionnels du territoire.

En marketing territorial, l'image revêt une importance capitale. Elle est un puissant levier de réussite des politiques territoriales qui vise à valoriser le patrimoine du territoire, ainsi qu'à diminuer le risque qui entoure la décision d'investissement. Et la mise en pratique du marketing territorial vise à mettre en place des outils, afin de convaincre les cibles dudit territoire et les inciter à y investir et à s'y installer.

Ces outils du marketing territorial basés sur l'écoute du terrain, s'appuient sur des programmes et des actions de promotion qui contribuent à l'attractivité territoriale et au développement des territoires. Il s'agit des projets d'infrastructures et des équipements de base, de la stratégie d'ambassadeurs qui permet de représenter le territoire, faire connaître ses atouts économiques et ses développements futurs et de développer son potentiel touristique. 
Par ailleurs, les outils du marketing territorial se construisent essentiellement pour différencier un territoire par rapport à ses concurrents, attirer plus d'entreprises mobiles et touristes ainsi qu'assurer un cadre de vie agréable aux habitants. Parmi ces outils, on trouve la veille territoriale qui vise une surveillance active de l'environnement qu'il soit économique, technologique ou de nouveaux entrant; et la marque territoriale qui est considérée également comme un atout stratégique pour augmenter la conscience et la fierté des habitants du territoire, et le promouvoir à l'échelle nationale et internationale, etc.

\subsubsection{La Veille Territoriale}

La veille territoriale a pour principal objectif d'observer de manière permanente et de détecter l'évolution de la situation des territoires concurrents; favoriser la découverte de nouvelles idées, les façons de faire et les méthodes de travail qui pourraient être appliquées facilement sur son propre territoire ; surveiller les actions marketing et publicitaires des concurrents ; examiner les données publiques relatives aux performances économiques des territoires concurrents ; et évaluer les offres territoriales des concurrents (points forts et points faibles), afin de prévenir les menaces qui proviennent de la concurrence et de saisir les opportunités qui vont rendre le territoire en question plus efficient. Il est à préciser que cette stratégie vise aussi à évaluer l'image et la compétitivité dudit territoire. D'ailleurs, la veille territoriale constitue un outil de benchmark, qui consiste à identifier un concurrent comme référence pour faire des comparaisons. Pour réussir cette stratégie, il faut tout d'abord définir les objectifs et les axes de surveillance ; définir le type d'information jugée utile ; identifier et sélectionner les sources d'informations ; collecter les informations nécessaires pour les traiter et les analyser par la suite et enfin effectuer une synthèse et exploiter l'information obtenue.

Il faut distinguer plusieurs types d'informations à surveiller et à collecter, notamment : - L'information blanche : ce type d'information est disponible et à libre disposition, sans fournir aucun effort, par exemple : les informations disponibles sur le site du concurrent, les forums, les vidéos sur Youtube, les articles de presse ; - L'information grise : ces informations nécessitent un effort pour les avoir, vu qu'ils ne sont pas disponibles pour tout le monde, mais tout en restant dans la légalité, à travers l'interrogation de ses partenaires, ses fournisseurs et ses clients, faire des entretiens d'embauches aux salariés de ses concurrents, les clients mystères ; - L'information noire : ce type d'information est obtenu de façon illégale (volées), notamment le piratage des serveurs,...

\subsubsection{La marque territoriale}

Pour Marion (2002) le rôle de la marque territoriale de la ville est de générer et de diffuser un univers de signification autour de "l'objet ville" : une sorte de machine à signification. La marque/ville est donc, d'une certaine manière, l'échafaudage de sens autour du territoire.

La marque territoriale est à la fois un dispositif d'organisation interne et un outil de promotion externe permettant d'exprimer son originalité. Sa création nécessite des dépenses importantes, mais ses retombées sont bénéfiques et donne lieu à un meilleur positionnement. La création d'une marque territoriale n'est pas obligatoire pour réussir, l'essentiel c'est que tous les acteurs territoriaux soient d'accord sur les attributs et valeurs qu'ils souhaitent promouvoir.

Pour qu'une marque trouve sa puissance, son utilité et sa réussite, il faut premiumiser le territoire dans l'esprit des cibles, le projet de territoire doit être exprimé par la marque territoriale, retrouver ses bases identitaires avant de contribuer à représenter l'imaginaire du territoire, susciter l'envie des cibles vu que la marque territoriale a pour objectif de rendre le territoire plus attractif à l'égard des cibles et non seulement à l'égard des responsables territoriaux, augmenter la visibilité et la notoriété du territoire. 
Selon Kapférer (2006), la marque est un gage de qualité et un repère pour le consommateur ; c'est en ce sens que la marque territoriale est un outil stratégique de différenciation, d'identification et d'association au territoire, ainsi qu'une aide pour développer le territoire de façon harmonieuse et internationale.

Une marque territoriale a pour objectif de promouvoir une identité forte afin de se distinguer dans le concert des territoires et ce, dans une économie mondialisée, être un vecteur de communication pour faire connaitre le territoire et ses produits par les outils classiques et modernes de diffusion (Férérol, 2013). La mise en place d'une stratégie de marque territoriale est destinée à atteindre plusieurs objectifs indissociables à savoir, communiquer sur les valeurs du territoire, attirer plus d'investisseurs, dynamiser l'économie locale, renforcer la fierté d'appartenance, accroître la quantité de sa production ce qui permet de faire des économies d'échelle, améliorer l'attractivité, la notoriété et l'image d'un territoire, offrir la possibilité d'associer une histoire et une personnalité à un produit capable de justifier un niveau de prix, promouvoir une identité forte afin de se distinguer par rapport aux autres territoires, s'ouvrir sur le monde, ainsi qu'être un vecteur de communication pour faire connaître le territoire et ses produits.

La marque territoriale créée à l'échelle d'une ville peut être globale (viser plusieurs marchés comme Be Berlin) ou spécifique à un segment de marché précis (les touristes à New-York avec I Love NY). De manière générale, la marque territoriale est un outil puissant au service du positionnement territorial et par conséquent, au service du marketing territorial vu qu'elle peut influencer la préférence pour un territoire.

\subsubsection{Ambassadeur territorial}

Les recommandations personnelles ont plus d'impact sur la qualité et l'expérience des visiteurs. De ce fait, les passionnés de leur territoire sont des puissants vecteurs de promotion territoriale, vu qu'ils constituent une véritable source d'information, de valorisation et qualification de son offre jouissant d'une grande crédibilité auprès des visiteurs. Par ailleurs, la stratégie d'ambassadeur territorial est créée dans le cadre du marketing territorial, elle est l'un des outils du marketing territorial visant à améliorer la promotion et l'attractivité du territoire sur plusieurs plans notamment, touristique, économique, social, sportif, culturel et commercial. Un ambassadeur est un individu qui joue le rôle de prescripteur en faisant la promotion du territoire. Cet ambassadeur rejoint un réseau qui est composé de personnes habitant dans le ou hors du territoire qui ont pour objectif principal la promotion et le rayonnement du territoire. En effet, l'évolution de simples consommateurs et de citoyens en de véritables ambassadeurs, à la fois porte-parole et conseillers du territoire, est devenue un processus indispensable pour gagner la fidélité des consommateurs et faire la différence. Un réseau d'ambassadeurs a des effets marketing bénéfiques, vu qu'en outre de sa contribution à établir un contact précieux entre les clients et les résidents, il a pour objectif de faire rayonner le territoire et de le faire connaitre. Grâce au réseau d'ambassadeurs, le territoire s'assure que ses produits et ses offres seront recommandés de manière plus crédible.

\subsection{Les facteurs clés de succès du Marketing Territorial}

Pour arriver à de bons résultats et pouvoir développer et promouvoir un territoire, il est impératif d'améliorer son infrastructure, accompagner et renforcer sa politique d'aménagement territorial, et il apparaît que la capacité à imaginer la cohérence des actions mises en place, le mode de gouvernance, l'organisation du partenariat et la mise en communication des projets sont également importants. 
Tout territoire se caractérise par un ensemble de ressources en lien avec son histoire et son contexte socio-spatial notamment, ses produits du terroir, ses paysages, son savoir-faire, ses patrimoines culturels qui le démarquent par rapport aux concurrents. La capacité de maitrise de ses propres ressources économiques pour une population ancrée dans un espace géographique donné, commence à s'affaiblir, à cause du fléau de la mondialisation et la concurrence élevée que connaissent les territoires.

L'histoire et la culture du territoire, le savoir-faire accumulé, le paysage, la qualification du capital humain, la stabilité économique et politique du pays constituent un ensemble de variables qui modifient et améliorent la valeur de la production locale. De ce fait, la définition et l'identification des ressources présentes localement sont nécessaire pour une meilleure valorisation du territoire. Dans le cas du marketing territorial, il est difficile de déterminer des critères de succès généralement pertinents, car chaque lieu doit décider lui-même de ce qu'il veut être ou devenir à l'avenir (Rainisto, 2003). De ce fait, le succès ou l'échec est fortement lié au territoire ou à la région elle-même et aux facteurs propres au pays (Jensen-Butler, 1997), ce qui rend l'évaluation de l'impact des stratégies marketing assez difficile.

Afin de réussir le marketing territorial, il est nécessaire de mettre le point sur certains facteurs clés tels que l'identité territoriale, la vision et le partenariat.

\subsubsection{L'identité territoriale}

Une identité territoriale est «un ensemble de valeurs, images, de concepts qui définissent la particularité locale, et l'existence d'un groupe par la délimitation d'un territoire » (Dubois, 1998). Elle fait référence à une population d'un territoire et la distingue des autres. Elle est la singularité d'un peuple d'une commune, d'un pays, d'un continent, ou d'une communauté de communes.

Cette quête d'identité apparaît être très importante dans le processus du marketing territorial et comme un axe principal dans la stratégie de promotion et de communication pour le territoire. De plus, la recherche d'identité créera surtout un véritable lien entre la population locale et les touristes.

Elle se traduit comme l'ensemble des traits culturels propres à un territoire qui le caractérise, le rend unique aux yeux des habitants comme à ceux des visiteurs, le différencie et peut définir sa personnalité. Autrement dit, l'identité se compose d'un patrimoine matériel incarné par une forme urbaine spécifique et un patrimoine immatériel composé de traditions, de réputations, de cultures locales et propres. L'histoire et l'expérience du territoire et une réelle base qui permettra de développer l'identité du territoire dans son avenir.

Il est impératif d'envisager au préalable l'identité du territoire avant de créer son image, sinon ils seront vides de sens et de valeurs. Cette image ne sera pas efficace et elle ne durera qu'un court temps, et elle captera la clientèle pour une brève période. L'identité et l'image territoriale s'impose de plus en plus aux territoires, afin de se démarquer et se distinguer, et permettront également de construire une stratégie globale tant en termes d'offres, que de communication en passant souvent par la construction de stratégie de marques.

\subsubsection{L'offre territoriale}

La promotion et l'attractivité d'un territoire sont conditionnées par un certain nombre de critères d'ordre environnemental, culturel, et social dont leur existence est nécessaire, notamment le territoire doit-être attractif, disposant d'une infrastructure facilitant l'implantation des entreprises et leur fonctionnement, et d'une main d'œuvre compétente et qualifiée ainsi qu'une bonne image. Le 
marketing territorial est une discipline où le territoire est vu comme un produit à vendre à des entreprises qui sont des clientes, et qui vont choisir dans une offre territoriale leur lieu futur d'implantation.

L'offre territoriale est un élément clé de différenciation pour attirer une activité économique dans un territoire. Une offre territoriale de qualité peut-être garantie par le respect des souhaits et des caractéristiques de la demande, à travers des études et des enquêtes territoriales. L'offre territoriale combine des caractéristiques nationales et internationales qui s'appliquent au territoire telle que la réglementation et la fiscalité ; des caractéristiques qui composent les attributs du territoire notamment physiques, géographiques et infrastructures; et des services d'accompagnement des acteurs économiques. L'offre territoriale se caractérise notamment, par le marché économique (concurrents, entreprises leaders,...) ; le bassin d'emploi (disponibilité du bassin d'emploi, degré d'implication au travail,...) ; le prix global (coût de la vie, subventions,...) ; l'immobilier (disponibilité,...) ; ressources naturelles ; la qualité de vie (qualité de l'équipement culturel, infrastructures de santé,...) ; la formation et la recherche (présence de pôles universitaires et de recherches,...) ; l'accessibilité (transports en commun, aéroports, logistique,...) ; la gouvernance (capacité de décision du territoire,...) ; l'image du territoire...etc.

Laurence Texier (1999) distingue ainsi trois grands types d'offre territoriale - qu'elle appelle offre d'implantation - dont le positionnement marketing diffère : - le «produit de ville» qui consiste en une offre de sites et qui correspond avant tout à des besoins fonctionnels ; - le produit «services ajoutés» qui prend en compte besoins fonctionnels et stratégiques: il implique, à la demande, d'autres acteurs que l'agence ou le service de développement pour la mobilisation de financements, de solutions de formation, de capacités de recherche... ; - le produit «attributs de territoire» essentiellement adapté à des besoins stratégiques émanant d'entreprises extérieures au territoire et qui nécessite la production d'informations justes et convaincantes sur les atouts du territoire, les facteurs-clés d'implantation, en bref une explicitation objective de l'attractivité territoriale.

\subsubsection{La vision et le partenariat}

Dans le cas du marketing territorial, la vision constitue une feuille de route indiquant l'objectif des autorités c'est-à-dire ce qu'elles veulent faire du territoire, et le niveau de développement qu'ils veulent atteindre, tout en définissant une orientation et une stratégie pour le développement et la promotion du territoire. Par ailleurs, la vision produit des stratégies et des objectifs concrêts.

Egalement, face aux défis que connait le monde, notamment économiques, sociaux et environnementaux, aucun territoire ne peut répondre seul aux nouveaux besoins. De ce fait, le partenariat entre les acteurs territoriaux s'avère primordial à la promotion et au développement territorial, vu qu'il permet d'inventer des solutions et de proposer des idées de promotion territoriale plus pertinentes. Cette démarche impose à une organisation ou à un individu de s'impliquer et de prendre les risques, au lieu d'être un simple acteur. Donc, Il s'agit de réfléchir et d'agir ensemble afin de trouver des solutions et des réponses plus adaptées aux besoins identifiés en s'appuyant sur les capacités de chaque acteur, et non pas seulement, de mettre à disposition du territoire des moyens. Ce partenariat entre les différents acteurs privés et publics peut contribuer au développement territorial à travers la satisfaction des besoins internes du territoire de façon plus efficace, la création de la valeur économique, ainsi qu'il a pour objectif de rendre le territoire plus performant et plus attractif vu que le partenariat entre les acteurs représente un facteur d'attractivité vis-à-vis de l'extérieur en améliorant le cadre et les conditions de vie des habitants du territoire. 
Cette démarche de partenariat, permettra également de renforcer l'identité du territoire ; assurer l'unicité du message autour des valeurs partagées ; développer des stratégies et des démarches plus innovantes en faveur de l'attractivité et du développement territorial ; valoriser les atouts du territoire ; améliorer l'image et la notoriété du territoire, etc.

\section{Marketing territorial comme outil de la promotion du territoire casablancais}

La pratique du marketing territorial s'avère primordial afin d'accompagner la stratégie marocaine de la régionalisation avancée qui vise à donner plus d'autonomie et de pouvoir aux territoires, notamment au niveau économique, ce qui crée une concurrence entre les différents territoires marocains. Comme étant un outil de promotion et d'attractivité territoriale, plusieurs territoires marocains sont devenus conscients de l'utilité du marketing territoriale et ils ont commencé à le pratiquer, notamment le territoire de Casablanca.

\subsection{Présentation du territoire casablancais}

Le territoire casablancais est considéré comme un lieu d'innovation, ainsi que son patrimoine architectural représente un potentiel touristique. Il est le plus riche du Royaume, grâce à ses potentialités économiques susceptibles de relancer son développement territorial, et lui permettant d'être une destination privilégiée pour les hommes d'affaires désireux investir dans les secteurs vitaux de l'économie.

Casablanca se positionne comme un hub financier international qui regorge de potentialités économiques, industrielles et humaines à exploiter, et attire les investisseurs étrangers. Il est un territoire offrant des conditions nécessaires au développement et au rayonnement des entreprises.

\subsubsection{Les potentialités de Casablanca}

Ce territoire bénéficie d'une panoplie d'atouts notamment une destination privilégiée, alliant proximité, logistique, disponibilité de compétences et d'une main d'œuvre ainsi que des plateformes d'accueil aux normes internationales, qui séduisent plusieurs sociétés représentant plus de $80 \%$ du tissu industriel aéronautique national dans différentes spécialités tels que le câblage, la mécanique, l'assemblage mécanique, etc. Et qui font de ce territoire une destination privilégiée de la sous-traitance aéronautique. Par ailleurs, le territoire casablancais attire les investissements directs étrangers, et participe un peu près de la moitié dans la production nationale, en plus de ses universités qui participent au développement de la recherche scientifique et des compétences et des qualifications de ses jeunes diplômés.

Le territoire casablancais regroupe près de la moitié de la production industrielle du pays, grâce à la richesse et la diversification de l'offre de ses zones industrielles. L'industrie aéronautique dispose d'une zone franche permettant aux industries exportatrices de bénéficier de plusieurs avantages fiscaux, douaniers ou en ressources humaines. Egalement, la technopole de Nouaceur Midparc qui comporte déjà un certain nombre d'entreprises aéronautique, et des filiales de grands groupes internationaux, est une zone offrant tous les atouts pour s'y installer.

Selon une étude du Cabinet spécialisé en business research Infomineo, Casablanca prend la troisième position des territoires les plus attractifs pour les investissements en Afrique et dans le Moyen-Orient, vu que c'est la première place financière d'Afrique et hub continental qui dispose de nombreux arguments pour séduire les investisseurs à savoir, une position géographique exceptionnelle vu que Casablanca est située sur la carte du monde avec des racines en Afrique et le regard rivé sur l'Europe, l'Amérique et le Moyen-Orient et une stabilité politique reconnue.

Ce classement a été attribué par différents organismes qui marquent la reconnaissance des capacités de financement importantes de la capitale économique, ainsi qu'elle offre aux investisseurs un cadre très 
attractif pour le financement de leurs activités industrielles et de services ; des coûts très compétitifs et un climat des affaires attractif qui favorise l'activité économique à travers notamment la simplification des procédures administratives aux entreprises, un système de droit des affaires renforcé respectant la propriété industrielle et intellectuelle ainsi que l'amélioration de la transparence réglementaire, ce qui a motivé de nombreux groupes nationaux et internationaux à installer leurs sièges, leurs bureaux et leurs usines pour profiter de nombreux avantages; une plateforme orientée export ouverte sur l'international et un écosystème structuré au service des investisseurs afin d'accélérer les démarches de création d'entreprise, et leur assister dans leur recherche de partenariat ou d'obtention d'un statut fiscal particulier, de facilités de change et de simplification de procédures.

De ce fait, et selon le classement établi par Fortune 500s, le territoire Casablancais a réussi en 2016 à attirer 37 entreprises mondiales sur son sol contre 13 en 2015. En pourcentage, 17\% des 500 meilleures entreprises avaient une antenne régionale à Casablanca à la fin de 2016.

\subsubsection{Terrain de l'étude}

Il est nécessaire de préciser le terrain de l'étude empirique de ce travail de recherche à savoir le territoire casablancais. Et pour pouvoir répondre à notre problématique, pouvoir situer le territoire de Casablanca, et pouvoir trouver la relation qui existe entre le marketing territorial et la promotion du territoire de Casablanca, il s'avère important de procéder à un entretien approfondi-cible adressé à l'ensemble des autorités compétentes chargés du développement et de la promotion de ce territoire, à savoir la Commune Urbaine, le Centre Régional d'Investissement, Casa-Events et Casa Transport.

Afin de tirer des conclusions sur le degré d'utilisation du marketing territorial, les stratégies mises en place, sa contribution dans la promotion du territoire casablancais, ce paragraphe traitera l'analyse des réponses du guide d'entretien à travers l'outil Nvivo, et mettra le point également sur les actions et les retombées de la pratique du marketing territorial qui ont été dégagées de l'analyse.

\subsection{Analyse et résultats}

Il s'avère nécessaire d'analyser les réponses des guides d'entretien adressés aux différents acteurs territoriaux tels que la commune urbaine, Casa Events, Casa Transport et le Centre Régional d'Investissement afin d'en ressortir des résultats fiables et bien détaillés sur la pratique du marketing territorial, et de procéder aux conclusions de cette étude et répondre à la problématique de ce travail de recherche.

\subsubsection{Analyse et interprétation des résultats}

A ce stade une interprétation des résultats sera faite, sur la base des réponses du guide d'entretien.

\section{Nœud 1 : L'attractivité de la ville de Casablanca}

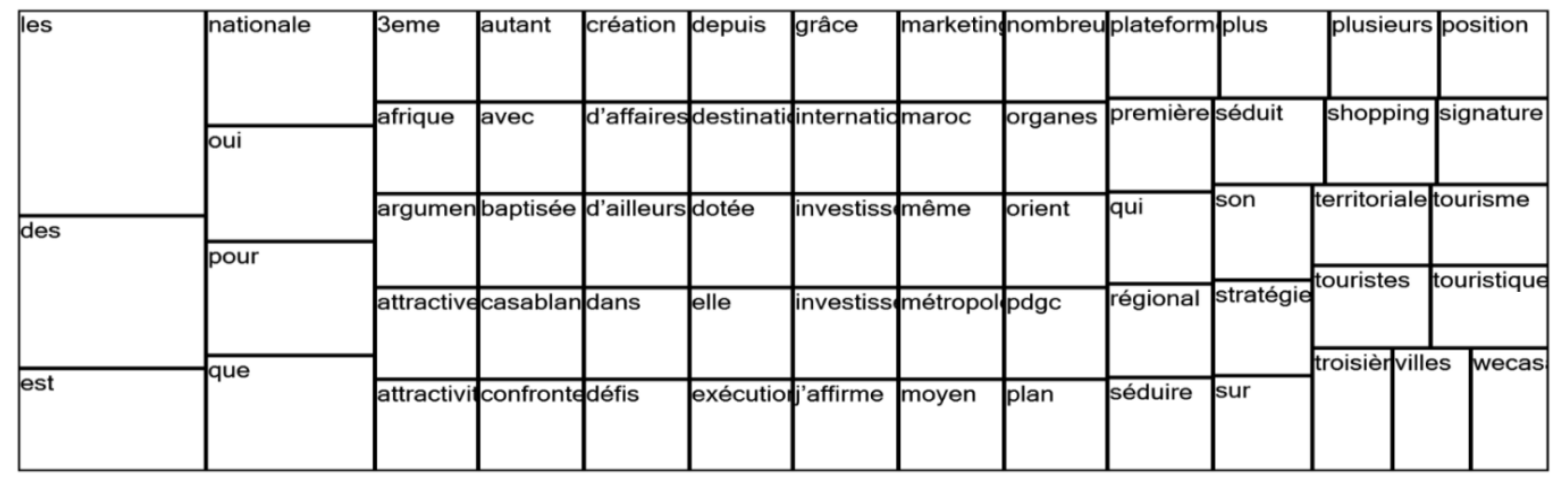


La métropole se positionne en tant que troisième ville des plus attractives pour les investissements en Afrique et dans le Moyen-Orient, et la 3ème destination touristique nationale avec le tourisme d'affaires.

\section{Nœud 2 : L'impact de Wecasablanca sur la promotion de Casablanca}

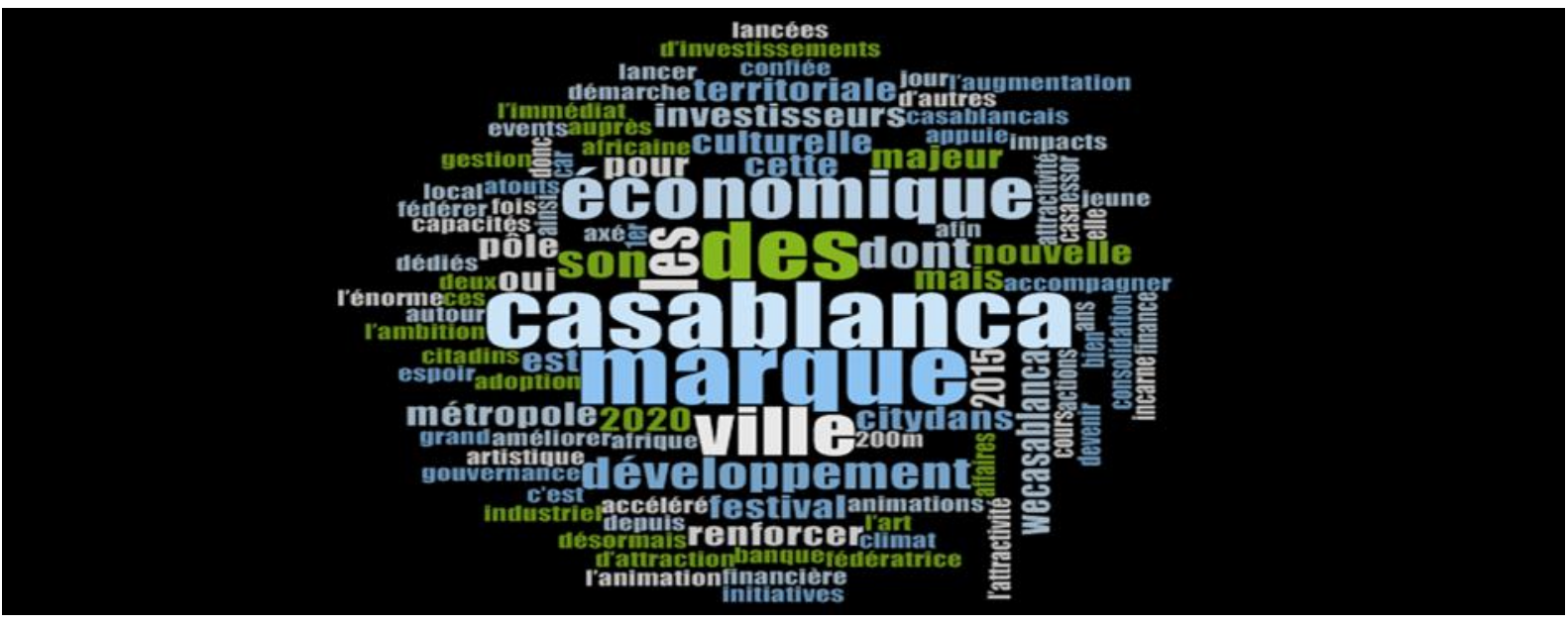

C'est une marque jeune et donc les impacts ne peuvent être mesurés dans l'immédiat, mais la démarche est fédératrice, et les initiatives lancées depuis son adoption montrent l'énorme potentiel dont regorge la métropole, ainsi que cette marque territoriale ne peut être que bénéfique dans la mesure où elle va permettre à la ville d'affirmer son identité, de renforcer la fierté d'appartenance des casablancais, et d'accélérer son essor économique. Depuis la mise en œuvre de la stratégie du marketing territorial, la ville de Casablanca est devenue un hub financier international connecté, inclusif et attractif. Par ailleurs, la banque mondiale a appuyé pour la première fois la ville pour améliorer sa gouvernance par un prêts de 200M\$.

La marque «Wecasablanca » vient renforcer la synergie entre toutes les parties prenantes afin de rayonner Casablanca à l'échelle nationale et internationale

\section{Nœud 3 : Les facteurs d'attractivité de Casablanca}

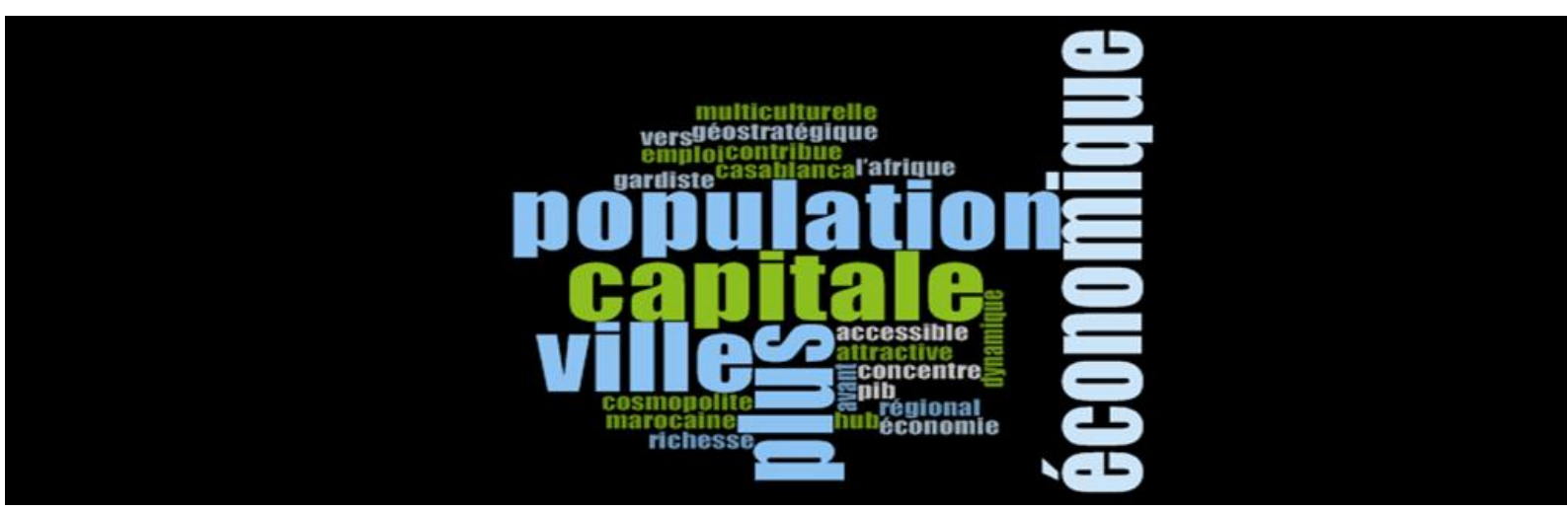

Casablanca est une ville attractive grâce à son modèle de smart city et aux initiatives qui ont accéléré son essor économique, à renforcer son attractivité et qui l'a rendu un espace urbain agréable à vivre. Elle est considérée également comme une destination privilégiée, alliant proximité, logistique, disponibilité de compétences et de main d'œuvre ainsi que des plateformes d'accueil aux normes internationales. 
Nœud 4 : Les freins qui empêchent la promotion de la ville de Casablanca

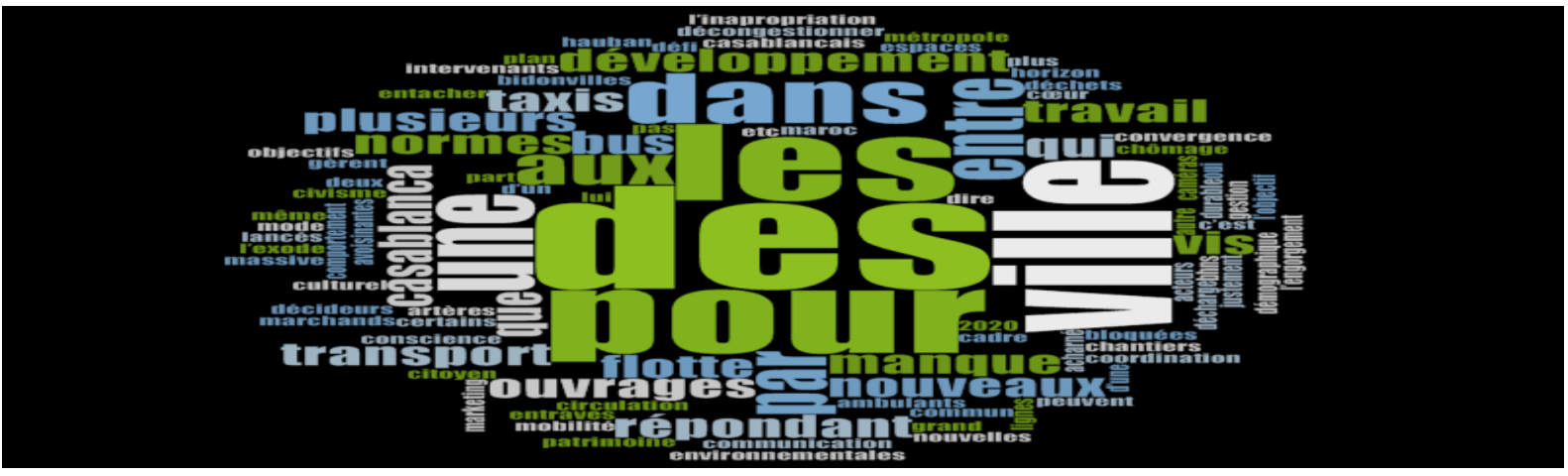

L'attractivité nationale et internationale de Casablanca la confronte à plusieurs défis tels que l'exode rural, l'habitat insalubre, un mode de transport et une flotte de taxis et bus ne répondant pas aux normes de grande ville, l'engorgement des artères principaux par une circulation souvent bloquées. De ce fait, plusieurs chantiers sont lancés pour remédier à ces entraves, notamment la rénovation de la flotte des taxis, les lignes de TRAM, les bus BHNS, etc.

\subsubsection{Les actions du marketing territorial appliquées à Casablanca}

Après 15 mois de réflexion et d'études, le territoire casablancais à créer sa propre marque territoriale afin de mettre en valeur et de distinguer son offre territoriale de celui proposée par d'autres territoires, renforcer la fierté d'appartenance à Casablanca, renforcer son identité culturelle, valoriser ses facteurs d'attractivité, faire du territoire casablancais une zone remarquable qui favorise l'implantation des entreprises et le développement des entreprises déjà implantées.

« Casablanca Events et Animations » s'insère dans la stratégie de développement de la métropole afin de promouvoir la nouvelle identité du territoire à travers des projets structurants, des événements sportifs, culturels et business en associant les différents acteurs du territoire casablancais et un dispositif de communication étudié tels que le site web disponible en cinq langues, proposant plus de 100 liens référencés et contient toutes les informations nécessaires ; le lancement de deux applications mobile à savoir "CasaBreak » permettant aux utilisateurs de trouver un commerce, un restaurant ou tout autre point d'intérêt en un seul clic, ainsi qu'il indique aux citadins et aux touristes les principaux lieux répertoriés en fonction de là où ils se trouvent, et « CasaMarathon » en vue de connecter tous les participants au dixième édition du Marathon International de Casablanca grâce à des filtres intelligents pour faciliter l'accès à l'information ainsi qu'inciter le public à participer à cet événement d'envergure ; La présence de Wecasablanca et de ses missions sur les réseaux sociaux pour créer la proximité avec les internautes et de faciliter la communication bilatérale; ainsi que la réalisation des vidéos et des films afin d'accompagner la promotion de l'attractivité globale et le dynamisme de le territoire, la faire découvrir ainsi qu'inciter les casablancais à devenir des ambassadeurs de leur territoire. Casablanca Events et Animations chargé de la promotion du territoire de Casablanca met en place plusieurs actions notamment, le salon smart city, le festival du shopping, le festival de l'art de Casablanca, un site web et un magazine dédié au territoire et bien d'autres actions qui redonne espoir dans l'animation culturelle, sportive, économique, artistique de Casablanca.... Casa- Events \& Animations a mis en place une stratégie de communication à $360^{\circ}$, à travers le lancement de compagnes de communication sur plusieurs supports notamment la télévision, radio, affichage, presse, web, ... ; une stratégie digitale intégrée, un écosystème digital intégrée ; la publication de supports d'édition notamment Magazine (Casamag), guide (Casa Shopping Guide), Bande Dessinée (Casawa) ; un parc d'affichage urbain 
propre ; programmes événementiels pour la révélation de la marque ; et la réalisation de plusieurs événements tels que Smart City Africa Expo, Fête de la musique, Marathon International de Casablanca ; ainsi que son jumelage avec plus de 50 villes au niveau international. Egalement, une marque fille a été mise en place à savoir «WECASABLANCA INVEST », portée par le Centre Régional d'Investissement de Casablanca-Settat depuis 2016. Cette nouvelle marque permet de donner une nouvelle identité visuelle, qui rime avec la nouvelle stratégie de communication que le Centre Régional d'Investissement a mis en place afin de renforcer ses actions de promotion et d'attirer des investissements porteurs et durables.

D'ailleurs le Centre Régional d'Investissement mène également des études stratégiques, de positionnement, et des stratégies de communication sur 3 ans, axée sur 2 objectifs principaux à savoir, concevoir avec les autorités territoriales et le gouvernement des offres territoriales sectorielles, et assurer la promotion des offres territoriales auprès des Investisseurs nationaux et étrangers. Le CRI organise et participe à plusieurs événements et conférences de promotion au niveau national et international, il assure également la réception de plusieurs délégations étrangères pour la présentation des opportunités d'investissements offertes par le territoire de Casablanca et par toute la région de Casablanca-Settat, l'organisation de Rendez-vous B to B, et l'utilisation du numérique comme vecteur de l'information notamment le nouveau portail web, nouvelle plateforme E-data relative à la région, des e-services...etc.

\subsubsection{Les retombées du marketing territorial sur le territoire de Casablanca}

Aujourd'hui, le territoire casablancais est la troisième destination touristique au Maroc après Agadir et Marrakech, vu que ses potentialités attirent le tourisme des affaires. Ainsi qu'il a abrité plusieurs congrès et rencontres internationales à caractère sportif, économique ou d'affaires. Autrement dit, ils lui permettent d'être une destination privilégiée pour les hommes d'affaires désireux s'investir dans des secteurs vitaux de l'économie. Sans oublier de signaler également que le secteur de la logistique, du commerce moderne, de l'offshoring avec les nouvelles technologies de l'information et de l'aéronautique sont également en plein développement. Selon la Direction Régionale du Tourisme, Casablanca se veut une destination d'affaires de choix en représentant $80 \%$ du total des arrivées à Casablanca. Ses potentialités économiques importantes sont susceptibles de relancer son développement territorial, ainsi qu'elle vit actuellement au rythme d'un ambitieux programme de développement et de projets d'envergure pour en faire un pôle financier international.

Grâce à la stratégie de la marque territoriale baptisée "Wecasblanca », la ville de Casablanca séduit autant les investisseurs que les touristes, elle a accéléré son essor économique, et à renforcer son attractivité.

Mais, la marque territoriale «Wecasablanca » est encore jeune et dont les impacts ne peuvent être mesurés dans l'immédiat et il est difficile de dresser un bilan pour deux ou trois années après le lancement, c'est un travail de longue haleine, qui doit-être fédérateur de toutes les forces vives et ses retombées seront mesurées après plusieurs années, mais la démarche est fédératrice.

\section{Conclusion}

Etre un territoire attractif et compétitif n'est pas une chose aisée. Certes, que les territoires sont soumis à une concurrence sans précédent dans l'histoire ce qui les conduit à lutter pour exister, attirer et retenir des habitants, lutter pour que les entreprises et les investisseurs les rejoignent, s'affirmer comme pôle touristique...etc., et c'est dans ce contexte qu'intervient le marketing territorial qui se présente comme une solution efficace en vue de mettre en avant les atouts et les forces d'un territoire qui cherche à renforcer son positionnement. 
Les résultats de notre recherche montrent que toutes les autorités sont conscientes du rôle du marketing territorial dans la promotion des territoires. De ce fait, plusieurs autorités territoriales disposent d'un service dédié au marketing territorial au sein de leur structure. Par ailleurs, la création d'une marque territoriale, l'utilisation de la communication territoriale, et la mise en place de plusieurs autres stratégies de marketing territorial, sont autant de stratégies qu'il s'avère nécessaire de mettre en place afin de pouvoir répondre aux objectifs de pérennité, de développement, et de promotion d'un territoire.

La limite majeure de ce travail et qui nous a empêché d'approfondir l'étude se situe dans la méthodologie de recherche, notamment la confidentialité des informations fournies par les autorités territoriales, la non-disponibilité des responsables, la non-disponibilité des informations chiffrées, vu qu'il aurait pu être intéressant d'avoir des données plus chiffrées pour dégager des résultats plus pertinents. Également, la marque territoriale de Casablanca est jeune, où les résultats ne peuvent pas être dégagés et quantifiés ce qui n'encourage pas encore les autres villes à y adopter. En outre, la mauvaise exploitation et visualisation de la marque territoriale «Wecasablanca » peut aussi affecter négativement son degré de réussite. Et donc, il serait lourd de revivre une expérience qui a échoué, sachant que son échec était à cause de la mauvaise mise en place et de l'insuffisance de communication et non pas de la création de la marque territoriale en elle-même.

\section{BIBLIOGRAPHIE}

[1] J. P. Aimetti, "Repères sur le marketing territorial", Oriental.ma, Rabat, pp. 20-21, 2013.

[2] H. Azouaoui, A. Ismaili, «L'identité de la marque de territoire et décision de localisation des entreprises : Approche par la littérature », Revue Marocaine de Recherche en Management et Marketing, Fès, pp. 114-123, 2015.

[3] A. Bendabdelhadi, S. Moussalim, « Le marketing territorial : outil de renforcement de la compétitivité et de l'attractivité des territoires-Cas de la région de l'Oriental ", Revue Marocaine d'Administration Locale et de Développement, 93, Rabat, 2012.

[4] «Casa Event », https://casainvest.ma/fr/cri/we-casablanca-invest, 15/10/2020.

[5] C. Chamard, L. Schlenker, «La place du marketing territorial dans le processus de transformation territoriale », Gestion et management public, Vol. 6(1), 2017.

[6] C. Chamard, «L'image de marque des territoires comme indicateur de leur performance : des enjeux pratiques aux interrogations éthiques? », IAE de PauBayonne, Laboratoire CREG, France, 2010.

[7] C. Chamard, « Le marketing territorial comment développer l'attractivité et l'hospitalité des territoires », De Boeck, Belgique, 2014.

[8] V. Dubois, « Politiques locales et enjeux culturels », La documentation française, Paris, 1998.

[9] A. El Khazzar, H. Echattabi, « les pratiques du marketing territorial dans le contexte marocain : Eléments de réflexion », International Journal of Innovation and Applied Studies, Vol $^{\circ}$ 16, 2016.

[10] M. Férérol, « Le développement économique en mode « Marque Territoriale » : l'exemple du Pays Basque Français », France, 2013.

[11] V. Gollain, « Le club des Développeurs Economiques d’lles de France, Réussir son marketing en 9 étapes », Ed. CDEIF, Paris, 2008.

[12] C. Jensen-Butler, "Compétition between cities, urban performance and the role of urban policy: a theoretical framework", In C., Jensen-Butler, A., Schachar and J. Vanweesep (Eds) European Cities in Competition, Aldershot : Avebury, 1997.

[13] JN. Kapferer, « La marque en question : réponses d’un spécialiste », Dunod, Paris, 2006.

[14] « Le Centre Régional d'Investissement de Casablanca-Settat », https://casainvest.ma/fr, 25/10/2020.

[15] G. Marion, « Lyon marque/ville internationale ?», 2002.

[16] S.K. Rainisto, "Sucess factors of place marketing : a study of place marketing practices in northern Europe and the Unites States », Helsinki University of Technology, 2003.

[17] Saône-et-loire, «stratégie de marque et d'attractivité du territoire », 4-55, 2011.

[18] TEXIER L., « Une clarification de l'offre d'implantation en marketing territorial : produit de ville et offre de territoire », Revue d'économie régionale et urbaine, no 5 : 1021-1036, 1999. 